NOTAS 



\title{
LAS RAÍCES INTELECTUALES DE LA ECONOMÍA EVOLUTIVA
}

\author{
ÓSCAR VARA CRESPO \\ Universidad Autónoma de Madrid \\ JORGE TURMO ARNAL \\ Universidad Autónoma de Madrid \\ ÁNGEL RODRÍGUEZ GARCÍA-BRAZALES \\ Universidad Autónoma de Madrid ${ }^{a}$
}

\section{INTRODUCCIÓN}

La economía evolutiva ha experimentado en los últimos años un notable auge en el ámbito científico europeo, desarrollando una amplia panoplia de modelos y teorías destinadas a estudiar diversos aspectos de los procesos económicos. Aunque la economía evolutiva se caracteriza como tal por utilizar los modelos poblacionales de la biología evolutiva ${ }^{1}$, adopta elementos cruciales de las obras de Schumpeter y Simon, lo que dota a su trabajo de una gran originalidad y profundidad analítica. La presente nota tiene por objeto examinar la estructura y alcance de esta escuela de pensamiento a través de sus referentes intelectuales, con el fin de evaluar el alcance de sus construcciones teóricas.

Los precursores en señalar la importancia de la biología para el análisis de los procesos económicos se remontan hasta Alfred Marshall ${ }^{2}$ y Thorstein Veblen ${ }^{3}$.

${ }^{a}$ Departamento de Análisis Económico. Carretera de Colmenar Viejo, Km.15, E-28049 Madrid. oscar.vara@uam.es; jorge.turmo@uam.es; angel.rodríguez@uam.es

${ }^{1}$ No consideramos aquí como economía evolutiva a toda la corriente que actualmente utiliza los juegos evolutivos como instrumento de modelización, dado que los autores que se consideran a sí mismos como integrantes de la economía evolutiva sólo utilizan modelos poblacionales.

${ }^{2}$ En el prefacio de sus Principles (1948, p. xiv), Marshall afirma: «The Mecca of economics lies in economic biology rather than economic mechanics. But biological conceptions are more complex than those in mechanics; a volume on foundations must therefore give a relatively large place to mechanical analogies; and frequent is made of the term equilibrium which suggests something of a statical analogy»

${ }^{3}$ En 1898 Veblen expuso sus ideas sobre la situación de la economía como ciencia en «Why is Economics not an Evolutionary Science?», artículo en el que afirmaba que, al no haber 
Marshall, aun siendo consciente de que la economía presentaba más analogías con la biología que con la mecánica, no desarrolló dicha analogía en el curso de su obra. Veblen, a pesar de sus críticas al panfisicalismo neoclásico, tampoco desarrolló de forma significativa sus ideas evolutivas en su pensamiento económico. Quienes realmente utilizaron la analogía biológico-evolutiva, aunque no hasta sus últimas consecuencias, fueron Schumpeter y Simon.

Sin embargo, el trabajo seminal de esta escuela lo constituye, sin duda, An Evolutionary Theory of Economic Change, de Nelson y Winter (1982). El punto de partida temático de Nelson y Winter es su descontento con las hipótesis utilizadas en los modelos neoclásicos tradicionales, modelos que, si bien resultan adecuados para explicar algunos fenómenos de la actividad económica, no lo son para el más significativo de entre ellos, precisamente la relación entre innovación y crecimiento.

La economía evolutiva presenta un marco de análisis alternativo y más amplio para tratar todo tipo de procesos económicos, por lo que constituye una opción en el estudio de fenómenos que presenten las siguientes características: por un lado, procesos sensibles a las condiciones iniciales, esto es, caracterizados por lo que se denomina «dependencia de la trayectoria». Por otro lado, situaciones en las que los agentes económicos no son homogéneos, sino que presentan comportamientos diferentes.

Este trabajo se estructura del siguiente modo: en primer lugar analizaremos los elementos teóricos que más han influido en la conformación de esta escuela de pensamiento, para pasar después a recoger algunas de las líneas de investigación actuales de esta escuela, siempre en relación con la tradición de la que parten, finalizando con un breve apartado de conclusiones.

\section{LAS BASES SOBRE LAS QUE SE ASIENTA EL MARCO TEÓRICO GENERAL DE LOS EVOLUTIVOS}

\subsection{Nota sobre la teoría evolutiva en biología}

En primer lugar, tendremos que detenernos brevemente a analizar la estructura conceptual de la biología evolutiva. Actualmente, ésta se articula alrededor de tres principios: variabilidad, herencia y selección natural. La variación individual de un organismo se produce mediante una mutación genética, como consecuencia de un «error» en el proceso de copia del ADN en los primeros momentos de la reproducción. Esta variación se hereda según las leyes de la genética, pero no

desarrollado Marshall la parte dinámica (evolutiva), la economía seguía siendo una ciencia retrasada, es decir, no «evolutiva». 
todas las variaciones heredadas sobreviven. La selección natural, operando a través del entorno -que incluye el medio físico, la presión de otras especies y la presión dentro de la especie- «escoge» aquellas variaciones más aptas y las incorpora al pool genético de la población. La mayor o menor aptitud (fitness) se mide por la mayor o menor capacidad para dejar descendencia. Las poblaciones más aptas -o que mejor consiguen adaptarse- son las que terminan sobreviviendo y las portadoras del cambio evolutivo. Este es, a grandes rasgos, el esquema conceptual de la biología evolutiva actual.

Aunque, históricamente, el primero en elaborar una teoría biológica evolutiva fue Lamarck ${ }^{4}$, el padre de la teoría evolutiva moderna es, sin lugar a dudas, Charles Darwin. Basándose en parte en elementos extraídos del pensamiento de Malthus $^{5}$, Darwin observó que, mientras los organismos se reproducen en progresión geométrica, las poblaciones de las especies permanecen estables a lo largo del tiempo; esto se explica, según este autor, por la existencia de un proceso de selección entre especies y dentro de las propias especies. En segundo lugar, observó también que las especies se caracterizan por la variación aleatoria de las propiedades específicas de cada uno de sus miembros, aleatoriedad que se ve restringida por el proceso de selección, que implica una adaptación al entorno. Sin embargo, y aun consiguiendo explicar el proceso de selección y adaptación, Darwin carecía de una teoría sobre la transmisión hereditaria de dichas variaciones. La explicación de la transmisión hereditaria de las propiedades genéticas la dio en 1866 Gregor Mendel. La síntesis entre ambas teorías se inició con los trabajos del biólogo alemán August Weismann y se ha venido en llamar neodarwinista -con este nombre aparece, por ejemplo, en Ayala (1994, p.38)-, constituyendo el núcleo de la actual mainstream dentro de la biología evolutiva.

Unos de los principales problemas conceptuales que tiene la biología evolutiva en estos momentos, y que se transmite también a la economía evolutiva, es el problema de la unidad de selección. Aunque existe un consenso mayoritario en que la unidad es el organismo individual, muchos autores no descartan que se produzca también a nivel de gen, grupo o especie: sobre esto, véanse, por ejemplo, Lewontin (1970), Hull (1980) y Sober (1996). En la economía evolutiva parece que existe también cierto consenso en considerar a las empresas como las unidades objeto de selección, aunque autores tan significativos como Metcalfe (1998, p. 27) no descartan que existan niveles más elementales.

\footnotetext{
${ }^{4}$ Como es bien sabido, el principio de la evolución lamarckiana es la herencia de los caracteres adquiridos. Este principio, reformulado para explicar la transmisión por imitación de los hábitos adquiridos, sigue teniendo vigencia en el pensamiento económico y social: véase Hayek (1997, pp. 214 ss.), y Nelson y Winter (1982, p. 11), pese a caer en desuso en la biología evolutiva desde hace bastante tiempo.

5 Sobre la influencia de Malthus en el pensamiento de Darwin, véanse Young (1969) y Bohlin (1991), en especial pp. 603-607.
} 


\subsection{Elementos tomados de la teoría económica}

«Our greatest intellectual debts are to Joseph Schumpeter and to Herbert Simon».

Nelson y Winter (1982, p. IX)

\section{Herbert Simon}

Las ideas de Simon se resumen en lo que él mismo denomina racionalidad limitada ${ }^{6}$. Según Simon, los agentes son racionales en tanto en cuanto persiguen sus objetivos con los medios más adecuados, pero no son maximizadores. Este autor ha mostrado que existen limitaciones objetivas a la capacidad de cálculo y toma en cuenta de hechos de los agentes. Los agentes están limitados tanto por el tiempo de que disponen como por su capacidad de computación de los hechos, que alcanza distintos grados según el agente, pero que nunca es completa. En consecuencia, los agentes han de servirse de una serie de procedimientos y reglas de actuación para tomar las decisiones. Y eso es lo que hacen las empresas como agentes.

Para Simon estos procedimientos cristalizan en rutinas. Puestos en marcha para tratar con un problema concreto, si los procedimientos se muestran adecuados, si superan la prueba a la que el entorno los somete, se convierten en rutinas que permiten funcionar a las empresas sin necesidad de que éstas tengan que dedicar mucho tiempo cada vez que tienen que tomar una decisión. Tal y como dicen Nelson y Winter (1982, p. 36):

"We base our modelling on the proposition that in the short and medium run the behavior of firms can be explained in terms of relatively simple decision rules and procedures».

Además, la economía evolutiva toma de Simon otra idea que está conectada con la anterior. Estas rutinas de búsqueda son de índole local, y no global. Los evolutivos lo adoptan como un principio de actuación de las empresas, porque les parece una muy buena descripción de la realidad. Por tanto, para los evolutivos las empresas son racionales, porque actúan con toda la información de que pueden disponer dada su situación en el mercado y sus rutinas de procesamiento y búsqueda.

El otro elemento que los evolutivos toman de Simon está relacionado con el anterior, aunque es distinto. Si los agentes se mueven en un entorno de raciona-

\footnotetext{
${ }^{6}$ Véase su obra con el título de Models of Bounded Rationality que aparece en la bibliografía.
} 
lidad limitada, la maximización de una función objetivo tal y como plantean los neoclásicos, es totalmente imposible.

¿ Qué hacen, pues, los agentes? La respuesta de Simon (1987, p. 244) es:

«Faced with a choice situation where is impossible to optimize, or where the computational cost of doing so seems burdensome, the decision maker may look for a satisfactory, rather than an optimal, alternative».

El objetivo es un nivel determinado de la variable que se considera en cada caso. Cuando hablamos de empresas, esa variable ha de ser el beneficio, desde luego. La empresa busca conseguir un nivel de beneficio que se ha marcado como objetivo porque lo considera satisfactorio. Si el criterio se consigue, la empresa está satisfecha y por tanto no tiene acicate a cambiar, mientras que, si no se consigue, la empresa ha de actuar para conseguirlo. Para los evolutivos, la regla de actuación de los agentes es siempre la misma: dado un nivel satisfactorio de la variable objetivo, si se consigue, no hay incentivos para modificar nada; si no se consigue, es necesario introducir cambios.

Joseph A. Schumpeter

«[...] the term 'neoSchumpeterian' would be as appropriate a designation for our entire approach as 'evolutionary'»

Nelson y Winter (1982, p. 39)

Los economistas evolutivos basan buena parte de su análisis en la analogía entre los procesos de la actividad económica y los procesos biológicos. Al hacerlo así, coinciden con Schumpeter. En sus obras, Schumpeter deja claro que la actividad económica, si con algo tiene similitud, no es con la mecánica clásica, en la que un conjunto de cuerpos con posiciones y velocidades determinadas interactúan y conducen al sistema de una situación a otra, sino con la Biología7.

En el caso de Schumpeter, el agente fundamental es el empresario ${ }^{8}$ : él es el motor y autor del desenvolvimiento económico, por usar la expresión que empleó en su día su traductor al español. Los evolutivos, al analizar el comportamiento de los agentes económicos, se hacen eco del pensamiento schumpeteriano. La dife-

${ }^{7}$ En todas ellas hay referencias indirectas, pero el planteamiento más explícito lo realiza en la Teoría del Desenvolvimiento Económico (1967).

${ }^{8}$ Las referencias al empresario son constantes en la obra de Schumpeter. Para una descripción muy amplia del «empresario heroico» y sus acciones, conviene consultar sobre todo Schumpeter (1967), mientras que, para la actuación de las grandes empresas, de lo que él llama capitalismo trustificado, es mejor Schumpeter (1939) y sobre todo Schumpeter (1968). 
rencia es que los evolutivos consideran a la empresa, y no al empresario, como el agente. Los evolutivos construyen todo un marco general de análisis de los procesos económicos, y para eso es necesario trabajar en el nivel de las empresas. Además, los evolutivos, en línea con lo que hace la biología, no piensan en términos de individuos, sino de poblaciones en su conjunto, que constituyen por tanto su objeto de estudio. Sus poblaciones lo son de empresas.

Veamos ahora lo que hace referencia al entorno en el que actúan los agentes y sus efectos sobre éstos. Para Schumpeter, lo esencial es que el entorno es cambiante. Su objetivo es explicar los procesos de desarrollo de la economía, tal y como él los entiende. Para ello identifica las famosas cinco vías de cambio -Schumpeter (1967, p. 77)- y propone considerar al empresario individual como el gran agente del cambio, como el portador del mismo. El cambio en el entorno económico es una consecuencia directa de la actuación de los empresarios en lo que él denomina «destrucción creativa». Al introducir los cambios por cualquiera de las cinco vías, los empresarios destruyen buena parte de las combinaciones y la actividad económica existente, para reemplazarla por algo nuevo. En este punto, el rasgo fundamental que comparten los evolutivos y Schumpeter es que el entorno es dinámico. Como exponen Nelson y Winter (1982, p. 10):

"The broader connotations of evolutionary include a concern with processes of long-term and progressive change. The regularities observable in present reality are interpreted not as a solution to a static problem, but as the result that understandable dynamic processes have produced from known or plausibly conjectured conditions in the pas $t$-and also as features of the stage from which a quite different future will emerge by those same dynamic processes».

Sus afirmaciones las realizan explícitamente contra el modo de análisis usual en el marco neoclásico. Lo importante es que el equilibrio es sólo un punto en una trayectoria, que comprende un antes y un después. No se trata de que una perturbación saque a la economía del equilibrio y los procesos lo devuelvan a él. No es el equilibrio la situación más usual, sino un punto por el que la economía pasa sin detenerse.

Las consecuencias del cambio, la destrucción creativa para Schumpeter (1967, p. 161), son que:

«El empresario que alcanza el éxito se eleva socialmente, y con él su familia, que adquiere con los frutos de su éxito una posición que no depende de manera directa de la conducta personal».

El planteamiento de los evolutivos, también aquí y fundamentalmente aquí, es más amplio y detallado y, por tanto, la cuestión de los procesos de selección 
debidos a los éxitos o fracasos de los agentes es crucial. En palabras de Nelson y Winter (1982, p. 226),

«In a stylized Schumpetian evolutionary system, there is both a carrot and a stick to motivate firms to introduce better production methods or products. Better here has an unambiguous meaning: lower cost of production, or a new product that the consumers are willing to buy at a price above the cost».

Para los evolutivos, las empresas son seleccionadas por el entorno en el que se desenvuelven. Ese entorno es el que decide cuáles son las que mejor se adaptan y, por tanto, las que sobreviven. En muchos casos, el mercado es ese entorno, pero no en todos. Así, el entorno desempeña el mismo papel que el medio en el caso de la teoría de la evolución. La adaptación, la adecuación o no, al entorno, determina si el agente, en este caso la empresa, sobrevive, o si no lo hace. Selecciona, aunque no sea una selección ciega como la selección natural.

Este proceso de selección es el que los evolutivos aplican a la innovación. Para Schumpeter, el empresario innovador, mediante la destrucción creativa, desplaza a muchas otras empresas y estructuras. Con un planteamiento más desarrollado, los evolutivos dicen que las empresas innovan cuando, con sus actuales rutinas, no obtienen un nivel de beneficios que consideren satisfactorio ${ }^{9}$. En ese caso, cambian sus rutinas y buscan la innovación. Si el nivel de beneficios que obtienen es satisfactorio, no tienen incentivo alguno para innovar.

Si el cambio en las rutinas que implica la innovación se adecúa, se adapta bien al entorno económico en el que tiene lugar, el éxito llega en forma de beneficios elevados. Al hacerlo así, la empresa innovadora desplaza a otras que eran sus competidoras, en lo que constituye un mecanismo de selección, de manera que tan sólo sobreviven aquellas cuyas rutinas son más adecuadas al entorno.

\section{LÍNEAS DE ANÁLISIS Y DESARROLLO DE LA ECONOMÍA EVOLUTIVA}

Dentro de una enorme variedad de desarrollos, se identifican en la economía evolutiva tres grandes líneas de trabajo, asociadas a la obra de tres autores: Geoffrey Hodgson, Stanley Metcalfe y Ulrich Witt.

Hodgson es el autor más comprometido con el componente evolutivo y biológico de la escuela. Entiende que el uso de la analogía biológica dentro de la economía evolutiva debe entenderse no tanto como algo deseable en sí mismo, sino

\footnotetext{
${ }^{9}$ En el sentido que Simon le da al término, y que los evolutivos adoptan.
} 
como una reacción contra la corriente principal neoclásica ${ }^{10}$. Hodgson (1995, p. 354) hace de los hábitos -rutinas- e instituciones los «genes» de la evolución económica, dado que transmiten información y tienen un carácter lo suficientemente estable dentro del mundo social como para que sobrevivan más tiempo que los organismos -en este caso empresas o sociedades- en los que residen. Reconoce, eso sí, que hacer de los hábitos la unidad de selección, impide explicar satisfactoriamente la aparición de la novedad -la innovación.

Witt es quizá el autor más crítico en lo referente a la analogía con la biología evolutiva. En primer lugar, a diferencia de Hodgson y Metcalfe, Witt no ve tan claro cuál es la unidad objeto de la selección. Tampoco los conceptos de selección y replicación tienen, según Witt (2001, p. 46), un análogo claro en el terreno económico: los agentes económicos no se enfrentan a los procesos de selección natural de forma ciega, sino que son capaces de anticipar sus efectos ${ }^{11}$. Pero Witt no renuncia por ello a la importación de conceptos de la biología. Propone, por el contrario, estudiar los componentes genéticos de los procesos de formación del comportamiento económico ${ }^{12}$. Como es bien sabido, una parte esencial de esos procesos responde a las preferencias y a los deseos de los agentes, rasgos de las personas que, según Witt, la teoría económica ha tratado de forma insatisfactoria.

Stanley Metcalfe ha tratado de avanzar una solución al problema de las unidades de selección, identificándolo con un proceso de transformación de recursos en productos. Se pueden entender así las unidades de negocio como complejos de rutinas que tienen como objetivo la dirección de la actividad de la empresa, y son éstas -las rutinas- las que determinan el perfil competitivo de la unidad. Los procesos de mercado seleccionan así entre complejos de rutinas.

Metcalfe identifica tres tipos de cambios en el comportamiento de esta unidad de negocio: la innovación, la imitación por adopción y la difusión. La primera consiste en la aparición de nuevos tipos de comportamiento que se introducen o bien por la entrada de nuevas empresas, o bien por cambios en las empresas ya existentes. La imitación consiste en la copia de estas rutinas por otros agentes. La difusión es un proceso que se estudia a nivel de población y que se manifiesta en la aparición de diferenciales de crecimiento dentro de la misma.

${ }^{10}$ «La intención de este trabajo es tratar de utilizar la biología, tanto para ayudar a oponerse a la metáfora mecanicista, como para establecer una base que permita el desarrollo futuro de la ciencia económica». Hodgson (1995, p. 51.)

${ }^{11}$ «[...] an important part of the systematic changes observable in markets, both in the composition of participants and in their activities, may be attributed to the individuals' attempts to anticipate the effects of the market forces and to take account of them». Witt (2001, p. 48).

${ }^{12}$ «During human phylogeny, natural selection did shape human genetic endowments which, because those selection forces are no longer a source of systematic change, are still present an thus may have an impact on economic behavior». Ibidem. 
Pues bien, el proceso de selección opera sobre las nuevas unidades diferenciadas, con el resultado de permitir la continuidad de aquellas unidades que, por su complejo de rutinas de comportamiento, están mejor adaptadas, expulsando a aquellas que no consiguen superar las «presiones del entorno». Pero, para que esta adaptación se produzca, es esencial que las empresas, al igual que los organismos, sean capaces de replicar su capacidad de negocio, esto es, la estructura de rutinas y prácticas. De tal modo que, en el caso particular de la economía, las nuevas unidades de negocio deben ser las mismas que aquéllas de las que son réplicas. Es aquí donde aparece, según Metcalfe, el cambio. Las estructuras de rutinas se replican en el tiempo de forma no perfecta, esto es, aparecen comportamientos diferenciados como respuesta a las presiones del entorno. Este proceso, casi lamarckiano, debe asumirse para explicar la aparición de una innovación en parte aleatoria y en parte intencionada. Pero la estructura de la teoría evolutiva contemporánea no puede darle un acomodo explícito, no al menos si tiene que recurrir a mecanismos lamarckianos ${ }^{13}$.

\section{CONCLUSIONES}

Esta breve nota tenía como objetivo llamar la atención sobre una escuela de pensamiento que puede ser de gran utilidad para la investigación económica en general, y para la de historia económica en particular, en cuanto que constituye una reivindicación de que la historia importa. Sus raíces intelectuales, entre la biología evolutiva, la destrucción creativa de Schumpeter y la racionalidad limitada de Simon, hacen que se constituya en una línea de pensamiento de gran proyección en un futuro próximo. Esto no obsta para que tenga sus limitaciones -fundamentalmente el problema de la intencionalidad- que, por otro lado, son comunes a todo el mainstream dominante.

\section{BIBLIOGRAFÍA}

Ayala, F. (1994): La teoría de la evolución. Madrid: Ediciones Temas de Hoy.

Bohlin, I. (1991): «Robert M. Young and Darwin Historiography». Social Studies of Science 21 (4), pp. 597-648.

HAYEK, F. A. (1997): La fatal arrogancia. Madrid: Unión Editorial.

\footnotetext{
${ }^{13}$ «Technologies do not emerge fully fledged into the world, they develop painfully in a trail and error fashion, through sequences of related innovations which are very much responses to the experience of market developments. Whether this process of endogenous innovation is capable of being understanding in all but its broad outlines seems to me doubtful. But I would hate to dissuade anyone from trying». Metcalfe (1998, p. 7).
} 
Hodgson, G. (1995): Economía y Evolución. Madrid: Colegio de Economistas de MadridCeleste Ediciones.

Hull, D. (1980): «Individuality and Selection». Annual Review of Ecology and Systematics 11, pp.311-332.

Lewontin, R. C. (1970): «The units of selection». Annual Review of Ecology and Systematics 1, pp. 1-18.

Marshall, A. (1948): Principles of Economics. Nueva York: Mc Millan, octava edición. Metcalfe, S. (1998): Evolutionary Economics and Creative Destruction. Londres y Nueva York: Routledge.

Nelson, R. y WinTER, S. G., (1982): An evolutionary theory of economic change. Cambridge, MA: Harvard University Press.

Schumpeter, J. A. (1939): Business Cycles. Nueva York: McGraw Hill.

- (1967): Teoría del desenvolvimiento económico. México: Fondo de Cultura Económica.

- (1968): Capitalismo, Socialismo y Democracia. Madrid: Aguilar.

Simon, H. A (1982): Models of Bounded Rationality. Cambridge, MA: MIT Press, vols. 1 y 2.

- (1987) «Satisfacing», en The New Palgrave: A Dictionary of Economics. Londres: Mcmillan, pp 243-245.

SoBer, E. (1996): La Filosofía de la biología. Madrid: Alianza Editorial.

Veblen, T. (1898): «Why is Economics not an Evolutionary Science?». Quarterly Journal of Economics 12 (4), pp 373-397

WIтT, U. (2001) «Economics and Darwinism», en Evolutionary Controversies in Economics: A New Transdisciplinary Approach. Berlín: Springer.

Young, R. M. (1969): «Malthus and the Evolutionists: The Common Context of Biological and Social Theory». Past and Present 43, pp. 109-145. 